\title{
System for tracking femtometer resonance shifts of silicon photonics microring resonator by locking tunable laser
}

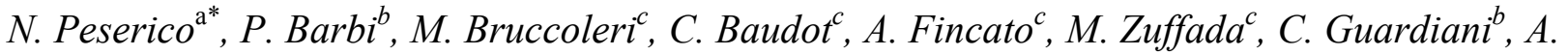 \\ Melloni $^{\text {a }}$ \\ ${ }^{a}$ Dip. Elettronica, Informatica e Bioingegneria, Politecnico di Milano, Milan, Italy; ${ }^{\mathrm{b}}$ Femtorays \\ Technologies, Rovereto, Italy; ${ }^{\mathrm{c}} \mathrm{STMicroelectronics,} \mathrm{Crolles,} \mathrm{France;}{ }^{\mathrm{d}}$ STMicroelectronics, \\ Cornaredo, Italy.
}

\begin{abstract}
Very fast and accurate frequency tracking is essential for many photonic integrated circuit applications, such as sensing or in telecommunication systems. In particular, accurately locking a laser frequency to micro-ring wavelength resonance shifts is crucial for many applications like for example sensing in general and bio-sensing in particular. In this work, we present an innovative system capable of locking a tunable laser frequency to a silicon photonics microring resonator (MRR) and of tracking any changes in its wavelength over time with very high speed, accuracy and sensitivity. An electronic digital feedback loop is used to balance the power at the drop/through outputs of the micro-ring thus finely tuning the wavelength of the input laser. The silicon photonics chip is equipped with integrated photodiodes at each port of the MRR. Thanks to the low noise characteristics of integrated photodiodes and the high resolution of the laser tuning capability it was possible to lock shifts of less than $7 \mathrm{fm}$ of residual noise at $1550 \mathrm{~nm}$. The digital implementation of the feedback loop allowed to reach bandwidths of up to $1 \mathrm{kHz}$. Fully functional samples of the locking system including different micro-ring resonators, with Q-factor varying from 5000 to 60000 were manufactured showing excellent performance results.
\end{abstract}

Keywords: silicon photonics, locking, microring resonator, feedback

\section{INTRODUCTION}

A significant and widespread growth of integrated silicon photonic systems and applications has been observed recently, leading to new devices in many different fields, such as telecommunications, sensors, and other. In particular this growth has been further enhanced by its compatibility with integrated CMOS fabrication process which allows to take advantage from fast, continuous innovation of IC devices [1]. This incredible progress has allowed to develop new and highly sophisticated photonic circuits, either implementing a large number of optical components [2], or allowing to integrate optical and electronic devices on a single chip [3] or both. Commercial products including silicon photonic devices are very common particularly in high speed data communications, achieving very high bandwidths [4].

In many of these silicon photonic applications it is possible to combine and leverage common concepts of traditional IC design to realize innovative applications; one of such very common concepts is the use of feedback and frequency/phase locking based tuning. In particular there are many photonic circuit applications that benefit from electronic feedback control in order to lock the optical response of the transfer function to external inputs [5]. For example, Cox et al. demonstrated the locking of a MRR using balanced homodyne locking scheme in a Mach-Zehnder configuration [6]. The Mach-Zehnder scheme makes circuit insensible to power fluctuation while keeping the MRR locked. Another example shows the possibility to lock DML sources to silicon photonics MRR's, allowing the multiplexing of 4 10Gbps channels [7]. In all these applications, optical circuits are locked to external sources, using heaters or pn junction, however it is also possible to do the opposite, i.e. to lock an external source to the optical circuit.

One example of this case is for optical sensing, where the optical circuit is used as a transducer of external factors, and in particular, a major application is in bio-sensing where the optical device is used to transduce bio-molecule's interaction into physical signals, for example into an optical quantity [8]. There are several examples in literature of silicon photonics biosensors using different circuits, such as Mach-Zehnder interferometers [9] and microring resonators [10].

* nicola.peserico@polimi.it; phone+39022399 8979; http://photonics.deib.polimi.it 
Most of these systems use an open-loop scheme to determine the presence of target molecules, most often by monitoring the micro-ring resonance wavelength shift occurring when a target analyte is captured by receptors near the waveguide. In fact, the technique that is most frequently used to determine the presence of such analyte molecule is to sweep a tunable laser wavelength over a certain range and acquire the resonance notch position. The main drawback of such open-loop techniques is that the resolution is intrinsically limited to few picometers. To overcome this limitation, new schemes have been proposed by using a feedback loop to track the resonance shift [11].

In this paper we present a feedback loop based micro-ring resonator sensor system implemented in an advanced $14 \mathrm{~nm}$ silicon photonic technology. A finely tunable laser is locked to add-drop, high Q-factor micro-ring resonators balancing the electrical signals at the two photo-diode (PD) output ports. To maximize the system performance, integrated photodiodes are used, together with a dedicated electronic circuit board to acquire the signals from the photonic chip and generate the control signal for the tunable laser. The overall system can be seen as a Frequency-Locked Loop, with adhoc PID circuit applied in the feedback loop. The feedback loop, together with the extremely high Q-factor, allows to the system to resolve wavelength shift in the order of femtometers, with a central wavelength of $1550 \mathrm{~nm}$.

This paper is divided into five sections. We start with this brief introduction followed by Section two, where we describe the conceptual system design, including the basic system working principle, its components, noise sources, and show some simulation results. The third section describes the silicon photonics chip and provides some details on the optical transfer function of the different MRR's. Section four presents the experimental results obtained with the proposed system implementation thus demonstrating the outstanding performances that were achieved. Finally in Section five we draw some conclusions.

\section{CONCEPT, NOISE SOURCES AND SIMULATION}

\subsection{Proposed system's working principle}

As explained in Section 1, the proposed system uses a frequency locked loop scheme to tune the input laser source in order to track the resonance wavelength of the MRR. To lock to the microring the add-drop outputs are combined into an error signal which is converted into a feed-back current to drive the DFB laser. Figure 1 shows the block diagram of the circuit and a schematic concept of the feedback loop.
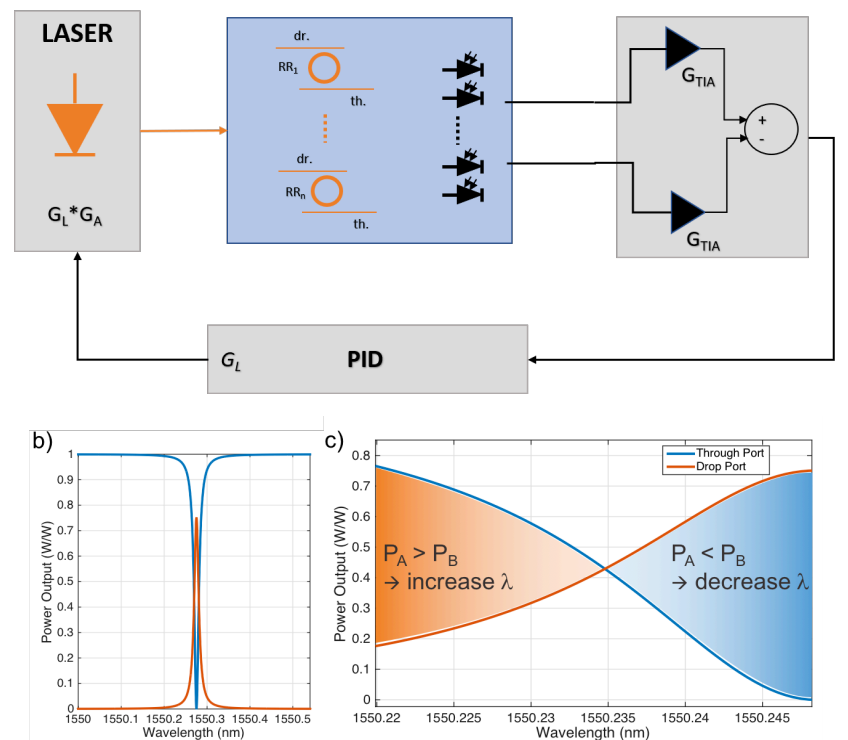

Figure 1: Block diagram and concept of the locking scheme. a) Block diagram of the system: a differential function of the optical outputs is used to feed the PID controlling the laser. b) Optical response of one add-drop microring resonator. c) Differential locking principle. 
By linearizing the optical response around the locking point, the entire system can be modeled by a linear transfer function, whose response to step change is exponential in time. The $\mathrm{G}_{\mathrm{s}}$ coefficient depends on the slope of the optical transfer function which is strictly related to the Q-factor of the MRR. Another important parameter is $G_{i}$, the integral coefficient of the PID block. By tuning $G_{i}$ it is possible to adjust the loop bandwidth. Other system parameters of interest are $\mathrm{G}_{\text {TIA }}$ (the transimpedance amplifiers gain), $\mathrm{G}_{\mathrm{L}}$ (the conversion factor of the laser controller), and $\mathrm{G}_{\mathrm{A}}$ (the conversion coefficient between laser current and its wavelength shift.

\subsection{Noise sources analysis}

The proposed system is subject to a number of different noise sources, and it is imperative to assess and minimize their impact in order to maximize the sensor's performance. First of all, the white noise of the photodiodes directly affects the resolution of the system. This noise source is minimized by using on-chip Ge photodiodes and limiting their bandwidth to $1 \mathrm{kHz}$. Another relevant noise source is the laser's linewidth jitter. This can only be optimized by selecting a high quality DFB laser with minimum linewidth. Chip-level thermal noise might be another potential source of noise, but since the time-scale of thermal fluctuations are in the order of seconds, it is possible to filter them out by using a highpass digital filter. Other optical device specific noise sources such as backscatter and nonlinearities, are also present but have a negligible impact on the system. It is important to emphasize that nonlinear effects are enhanced since a redshift of the microring induces an increase of the injected current in the laser which in turn causes the optical power output to increase, that reflects directly in the optical power circulating inside the cavity. This potentially positive feedback loop has to be limited by controlling the optical output power of the laser, with a suitable optical attenuator.

\subsection{Numerical simulations}

A 'Simulink model of the sensor system has been implemented to simulate the performance of the system and assess its potential optimal capabilities. The results for different level of photodiodes white noise and for different coupling factor of the microring are plotted in figure 2. Showing the standard deviation of the PID output as a function of wavelength. It is possible to see that femtometers resolution is achievable by carefully minimizing the noise, and that the coupling coefficients have a limited impact on the overall system's performance.

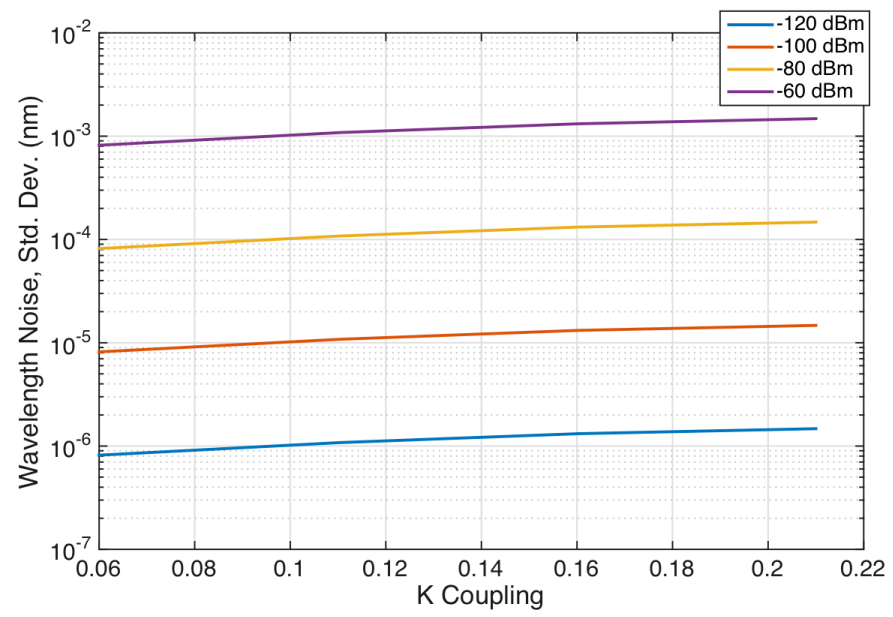

Figure 2: Simulated wavelength noise of the system, for different level of noise at the photodiodes and different coupling coefficient of the microring resonator. This last parameter shows limited impact on the performances, with respect to the noise level.

\section{SILICON PHOTONICS CHIP DESIGN AND CHARACTERIZATION}

The optical core of the system is the silicon photonic chip realized by STMicroelectronics [12]. The waveguide is rib shaped, whose dimensions are plotted in figure 3, together with the optical TE mode at $1550 \mathrm{~nm}$ wavelength.

The design of the chip provides 12 add-drop microring resonator equipped with integrated photodiodes at each output. Inputs of the microrings are performed by grating couplers. Another 4 microrings have been designed with grating couplers outputs in order to test them optically. To maximize the Q-factor, and so lowering the round-trip losses in the ring, all the microrings are perfectly circular rings, and the two couplers for each microring are designed with equal gap. 
a)

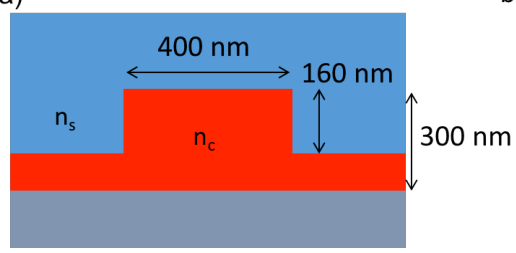

b)

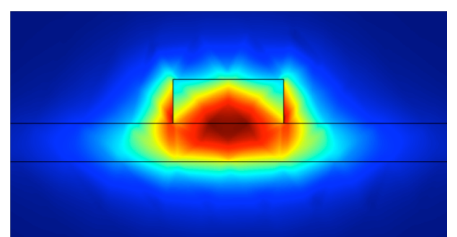

Figure 3: Optical waveguide of the silicon photonics chip. a) STMicroelectronics waveguide. Core material is silicon $\left(n_{c}=3.5\right)$, while surrounding material is silica $\left(n_{s}=1.45\right)$. b) The electrical normalized field intensity of the fundamental quasi-TE mode of the waveguide.

We optically tested the 4 microrings that have both optical inputs and outputs. The results are plotted in figure 4 . We highlight 2 main achievements of the chips: a low insertion loss, typically lower than $6 \mathrm{~dB}$, and the high Q-factor obtainable with the smallest coupling coefficient (so the largest gap between feed waveguide and ring waveguide). In particular, Q-factors higher than 60.000 are achieved without any ad-hoc fabrication step.
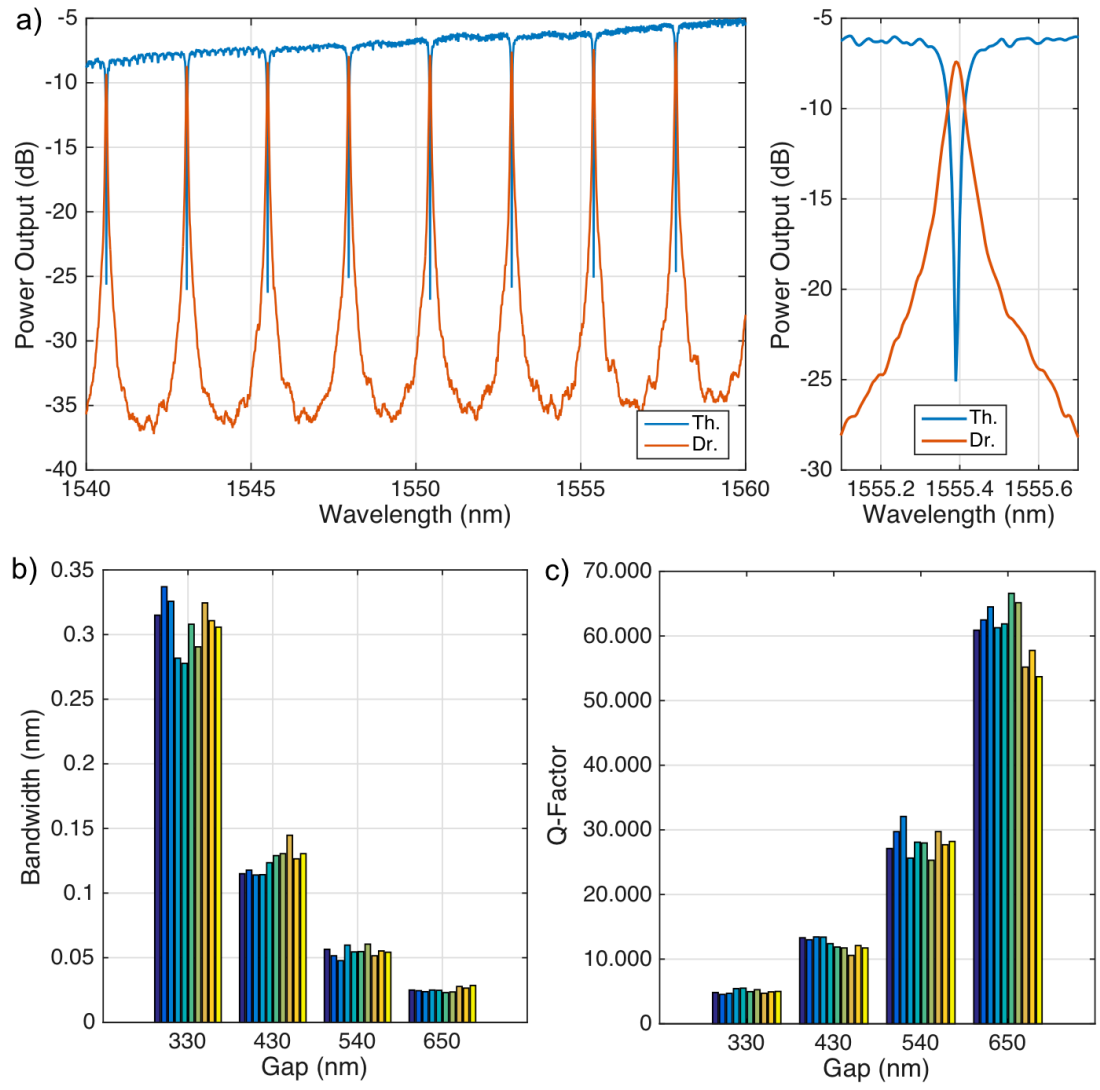

Figure 4: Measured optical characteristics of the silicon photonics microrings. a) Optical spectrum of the microring having $540 \mathrm{~nm}$ gap in the waveguide couplers. b) Optical bandwidth of 4 microrings tested on 10 different chips. c) Q-factor computed from the bandwidth, at $1550 \mathrm{~nm}$ wavelength.

The silicon photonics chips are then packaged into DIP socket to minimize interference and additional noises between photodiodes and the first stage of amplification (TIA amplifiers). 


\section{EXPERIMENTAL RESULTS}

A custom PCB design has been implemented integrating the TIAs and PID circuit alomng with proper signal multiplexing. In particular, the PID has been implemented by using a microprocessor following A2D conversion of the input signals. This allowed to easily control the $\mathrm{G}_{\mathrm{i}}$ parameter in real-time during the tests. A drawback of this choice however is that the resolution of the PID output is limited by the 16bit DAC between the PID and the laser controller. A photograph of the board and of the packaged chip are shown in figure 5. The laser is a JDS Uniphase laser for sensing application, with a linewidth of $700 \mathrm{kHz}$.
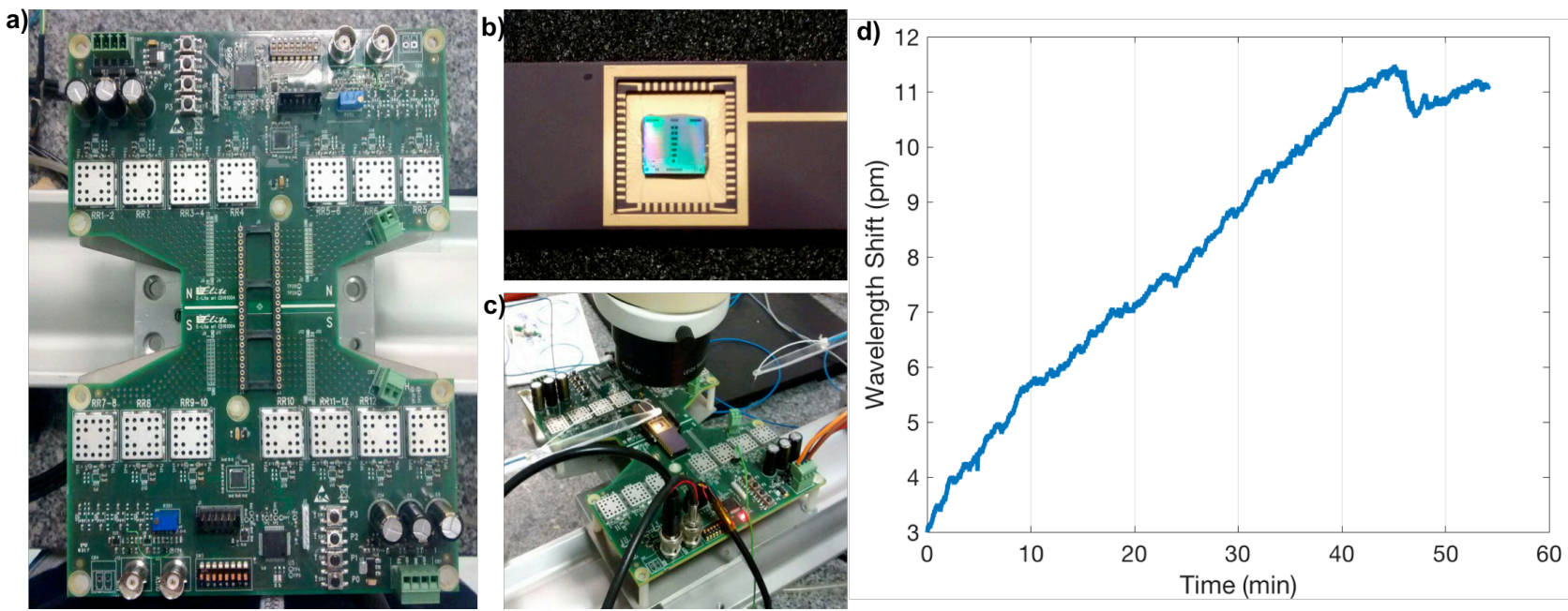

Figure 5: Picture and signal of the locking system. a) Electronic board implementing amplification and feedback stages. b) Silicon photonics chip packaged into a DIP48 carrier. c) Photo of the mounted platform with the input fiber in the left. d) Signal acquired during a locking experiment.

The data in figure $5 \mathrm{~d}$ show the value of the locking signal monitored over a $50 \mathrm{~min}$ time window; the linear trend is due to the heating of the chip. These results prove the stability of the proposed locking concept and. A comparison of the performance of different Q-factor MRR's at different optical power levels is presented in figure 6 .
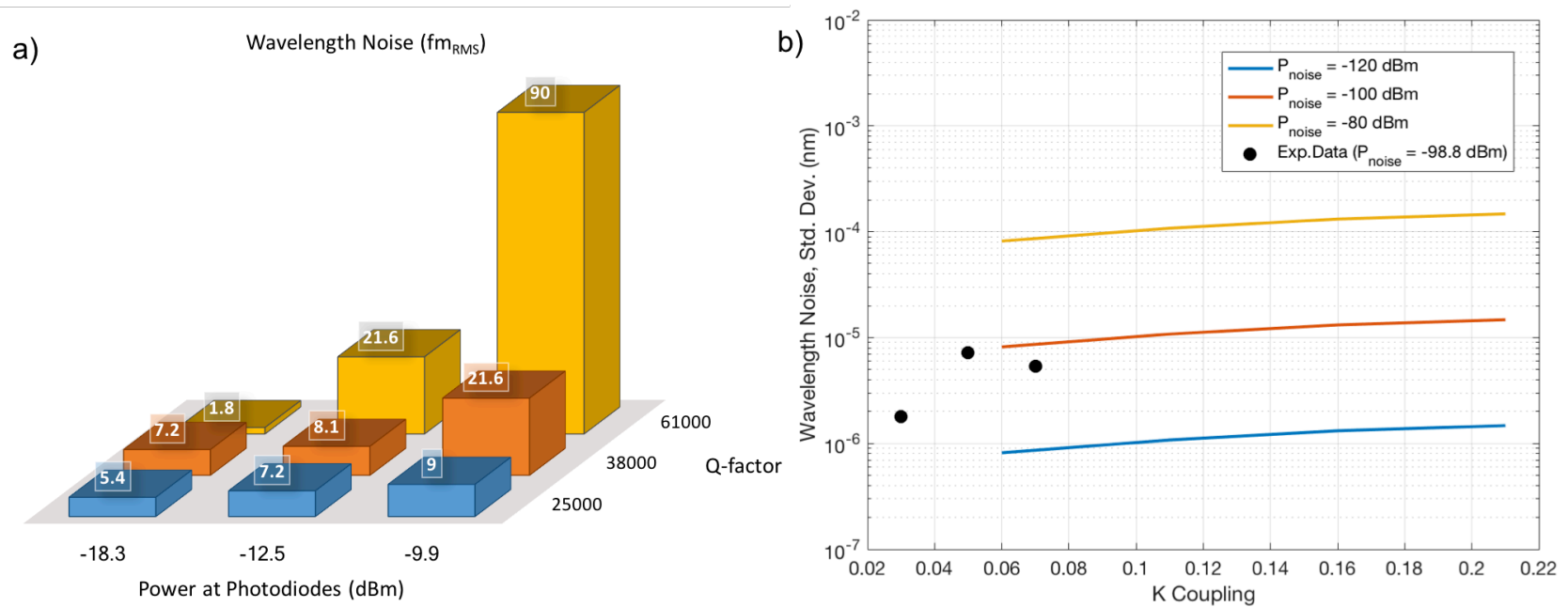

Figure 6: Measured performance of the locking system in terms of wavelength noise. a) Wavelength noise as $\mathrm{fm}_{\mathrm{RMS}}$ for 3 different microring resonators, varying the optical power circulating in the cavity. b) Wavelength noise chart comparing the experimental data for the lowest optical power with the simulation showed in figure 2. 
It is interesting to note some interesting trends. First of all by increasing the optical power a larger wavelength noise is induced. This is more evident at higher Q-factor values. This can be linked to nonlinear and thermal effects. Another trend that can be observed is the correlation between Q-factor and noise, at the lowest optical power. This was somewhat predicted by the simulation, even if the impact of the Q-factor is smaller than the one due to the photodiode's noise. The experimental results and the simulation data match quite well at low optical power levels as it can be seen in figure $6 \mathrm{~b}$.

\section{STEP DETECTION}

An important detection test has been performed by tracking changes to step perturbations in the resonance position. In particular, for label-free biosensors, the binding between analytes and receptors produces a step shift of the resonance. The same behavior appears in label-based biosensors, when labels bind to the analytes. The system presented in this paper is capable of detecting step changes down to $9 \mathrm{fm}$. We experimentally induced step variation in the feedback loop by adding voltage steps of different amplitude during the test. The results are plotted in figure 7 .
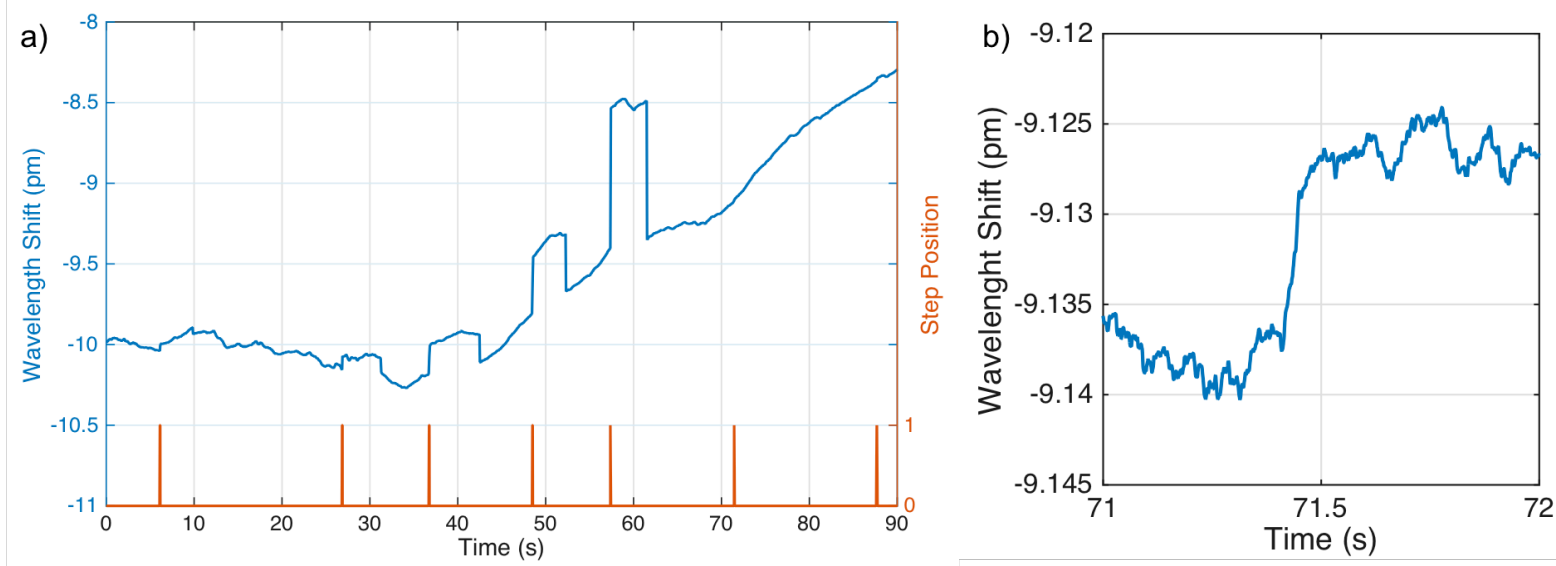

Figure 7: Step detection during locking experiment. a) Wavelength shift signal acquired during the experiment, together with the results from the step detection algorithm, using a numerical derivative of the signal. The induced steps have the following amplitudes: 45, 90, 180, 360, 900, 9, and 18 femtometers. b) Section of the wavelength shift signal during $9 \mathrm{fm}$ step.

From the acquired data, a numerical derivative of the signal is used as step detection algorithm to find the position of the various steps. In figure $7 \mathrm{~b}$, it is shown the smallest step of approximately 9 femtometers, that to the best of our knowledge represents the smallest step variation detected by a silicon photonics chip to date. This shift corresponds to $5.8 \cdot 10^{-9}$ RIU step change.

\section{CONCLUSIONS AND NEXT STEPS}

In this paper we presented the results obtained by applying frequency locking to a silicon photonic microring resonator. The results demonstrated the possibility of detecting resonance shifts in the femtometer range, down to a record 9 fm value for step changes similar to those typically occurring in optical biosensors when analyte bind to receptors. Further improvement of the system could be achieved by using an analog feedback loop and smaller linewidth lasers. We also intend to test on-chip integrated lasers as well as multiplexed MRR arrays. Finally we plan to apply this system to perform actual biosensing experiments in other to detect single molecule bindingin conjunction with magnetic labeling [13].

\section{REFERENCES}

[1] Soref, R. "The past, present, and future of silicon photonics." IEEE Journal of selected topics in quantum electronics 12.6: 1678-1687, (2006). 
[2] Annoni, A., Guglielmi, E., Carminati, M., Ferrari, G., Sampietro, M., Miller, D.A., Melloni, A. and Morichetti, F., 2017. "Unscrambling light—automatically undoing strong mixing between modes. " Light: Science \& Applications, 6(12), p.e 17110, (2017)

[3] Sun, C., Wade, M.T., Lee, Y., Orcutt, J.S., Alloatti, L., Georgas, M.S., Waterman, A.S., Shainline, J.M., Avizienis, R.R., Lin, S. and Moss, B.R., 2015. "Single-chip microprocessor that communicates directly using light. " Nature, 528(7583), pp.534-538, (2015)

[4] Rumley, S., Nikolova, D., Hendry, R., Li, Q., Calhoun, D. and Bergman, K. "Silicon photonics for exascale systems." Journal of Lightwave Technology, 33(3), pp.547-562, (2015).

[5] Morichetti, F., Grillanda, S., and Melloni, A. "Breakthroughs in photonics 2013: Toward feedback-controlled integrated photonics." IEEE Photonics Journal, 6(2):1-6, (2014)

[6] J. A. Cox, A. L. Lentine, D. C. Trotter, and A. L. Starbuck. "Control of integrated micro-resonator wavelength via balanced homodyne locking. " Optics express, 22(9):11279-11289, (2014).

[7] Grillanda, S., Ji, R., Morichetti, F., Carminati, M., Ferrari, G., Guglielmi, E., ... \& Vannucci, A. "Wavelength Locking of Silicon Photonics Multiplexer for DML-Based WDM Transmitter. " Journal of Lightwave Technology, 35(4), 607-614, (2017).

[8] Estevez, M.C., Alvarez, M. and Lechuga, L.M. "Integrated optical devices for lab-on-a-chip biosensing applications. " Laser \& Photonics Reviews, 6(4), pp.463-487, (2012).

[9] Densmore, A., Vachon, M., Xu, D.X., Janz, S., Ma, R., Li, Y.H., Lopinski, G., Delâge, A., Lapointe, J., Luebbert, C.C. and Liu, Q.Y., "Silicon photonic wire biosensor array for multiplexed real- time and label-free molecular detection. " Optics letters, 34(23):3598-3600, (2009).

[10] Peserico, N., Annoni, A., Varriale, A., D'Auria, S., Bellieres, L., Cuesta-Soto, F., Rodrigo, M., Peransi, S. and Melloni, A. "Experimental demonstration of integrated photonic free-label biosensor for CBRN threats using micro-ring resonators." In Transparent Optical Networks (ICTON), 2016 18th International Conference on, pp. 1-4. IEEE, (2016).

[11] Su, J., Goldberg, A.F. and Stoltz, B.M., "Label-free detection of single nanoparticles and biological molecules using microtoroid optical resonators." Light: Science and Applications, 5(1): e16001, (2016).

[12] Baudot, C., Fédéli, J.M., Marris-Morini, D., Caire-Remonnay, B., Virot, L., Olivier, S., Myko, A., Grosse, P., Grand, G., Bakir, B.B. and Hartmann, J.M. "Introducing photonic devices for $40 \mathrm{gbits} / \mathrm{s}$ wavelength division multiplexing transceivers on 300-mm soi wafers using cmos processes. " In Integrated Optics: Devices, Materials, and Technologies XVIII, volume 8988, page 89880O. International Society for Optics and Photonics, (2014).

[13] Peserico, N., Pratim Sharma, P., Belloni, A., Damin, F., Chiari, M., Bertacco, R. and Melloni, A. "Enhancement of integrated photonic biosensing by magnetic controlled nano-particles", SPIE Photonic West, (2018). 